ARTIGO ORIGINAL

\title{
Validação de um modelo completo em nível de árvore individual para uma floresta estacional semidecidual
}

\author{
Validation of complete individual tree model for a seasonal \\ semideciduous forest
}

Renato Vinícius Oliveira Castro ${ }^{1}$ (D), Carlos Pedro Boechat Soares ${ }^{2}$ (D), Helio Garcia Leite ${ }^{2}$ (D), Agostinho Lopes de Souza ${ }^{2}$ (D), Fabrina Bolzan Martins ${ }^{3}$ (1) , Gilciano Saraiva Nogueira ${ }^{4}$ (D), Márcio Leles Romarco de Oliveira ${ }^{4}$ (i)

${ }^{1}$ Universidade Federal de São João del-Rei - UFSJ, Sete Lagoas, MG, Brasil

${ }^{2}$ Universidade Federal de Viçosa - UFV, Viçosa, MG, Brasil

${ }^{3}$ Universidade Federal de Itajubá - UNIFEI, Itajubá, MG, Brasil

${ }^{4}$ Universidade Federal dos Vales do Jequitinhonha e Mucuri - UFVJM, Diamantina, MG, Brasil

Como citar: Castro, R. V. O., Soares, C. P. B., Leite, H. G., Souza, A. L., Martins, F. B., Nogueira, G. S., \& Oliveira, M. L. R. (2020). Validação de um modelo completo em nível de árvore individual para uma floresta estacional semidecidual. Scientia Forestalis, 48(126), e3061. https://doi.org/10.18671/scifor.v48n126.10

\begin{abstract}
Resumo
O objetivo deste estudo foi validar um modelo completo em nível de árvore individual desenvolvido para uma Floresta Estacional Semidecidual em estágio médio de sucessão. Foi realizada uma simulação com o objetivo de interagir os submodelos de mortalidade, crescimento e ingresso, os quais compõem um modelo completo em nível de árvore individual para florestas naturais. Uma rede neural artificial (RNA) foi previamente selecionada para compor o submodelo de mortalidade, nas quais realizou a previsão da probabilidade de mortalidade para cada indivíduo entre dois períodos de medição. Outra RNA foi selecionada para projeção do dap, altura total e altura do fuste, simultaneamente, para um ano de medição futuro. Já para o submodelo de ingresso, foi determinada uma taxa periódica média de ingresso de árvores entre cada período de medição. Para a validação do modelo foram utilizados dados de quatro parcelas monitoradas entre os anos de 1994 e 2008, sendo essas parcelas independentes das utilizadas para gerar os submodelos. A partir da medição do ano de 1994, realizou-se a simulação de mortalidade, crescimento e ingresso para os anos de 1997, 2000, 2004 e 2008. Os resultados da simulação foram comparados aos valores observados no ano final da projeção (2008). O modelo completo em nível de árvore individual foi eficiente na simulação do crescimento e produção florestal em todos os níveis de detalhamento. Os modelos em nível de árvore individual apresentam potencial de utilização de em florestas naturais estruturalmente complexas como a Floresta Estacional Semidecidual Montana em estágio médio de sucessão ecológica.
\end{abstract}

Palavras-chave: Floresta inequiânea; Redes neurais artificiais; Crescimento arbóreo.

\begin{abstract}
This research's goal was validate the individual tree model developed for a Seasonal Semideciduous Forest in middle succession stage. A simulation was executed aiming to integrate the mortality, growth and ingrowths sub-models, which are part of the individual tree level complete model for natural forests. A neural artificial network (RNA) was selected to integrate the mortality sub-model, which helped to predict mortality probability for each specimen between measuring periods. Another RNA was selected to project the $d b h$, total height and bole height, simultaneously, in a future measuring year. As for the ingrowths sub-model, an average tax of trees' ingrowths was determined between each measuring
\end{abstract}

Fonte de financiamento: CNPq (Processos 140334/2011-4 e 300941/2016-1).

Conflito de interesse: Nada a declarar.

Autor correspondente: renatocastro@ufsj.edu.br

Recebido: 24 agosto 2018.

Aceito: 10 julho 2019

Editor: Francides Gomes Silva Júnior.

(c) (1) Este é um artigo publicado em acesso aberto (Open Access) sob a licença Creative Commons Attribution, que permite uso, distribuição e reprodução cc) em qualquer meio, sem restrições desde que o trabalho original seja corretamente citado. 
period. For the model validation was used data from plots between 1994 and 2008; these plots were independent of the ones used to generate the sub-models. Since the measuring in 1994, simulations of mortality, growth and ingrowths were done in 1997, 2000, 2004 e 2008. The results were compared to the observed values in the final projection year (2008). The complete individual tree model was efficient in simulating growth and forest production in all details levels. The results confirm the potential use of individual tree models in structurally complex natural forests, such as the Montane Seasonal Semideciduous in middle stage of ecological succession.

Keywords: Uneven-aged forest; Artificial neural networks; Tree growth.

\section{INTRODUÇÃO}

O Bioma Mata Atlântica, que abriga uma vasta diversidade biológica e um alto grau de endemismo, estendia-se, originalmente, por uma área estimada de 1,1 milhões de $\mathrm{km}^{2}$ no Brasil (Shimizu, 2007; Brasil, 2012). Este Bioma, em Minas Gerais, totalizava 46\% do território do estado, mas atualmente encontra-se drasticamente alterado, e em sua maioria, por fragmentos de florestas secundárias, com apenas 10\% de área remanescente (SOS Mata Atlântica, 2012).

As florestas remanescentes da Mata Atlântica são fontes muito importantes de produtos madeireiros de alto valor agregado, além de conservar a diversidade biológica. Essas são compostas por um grande número de espécies, com as mais diferentes características silviculturais, ecológicas e tecnológicas (Scolforo et al., 1996; Rossi et al., 2007).

Estimar com precisão a dinâmica de crescimento, mortalidade e ingresso das florestas é um desafio constante dos pesquisadores florestais. Para isso, são utilizados os modelos de crescimento e produção, que permitem simular a dinâmica natural destas florestas, de modo a prever sua produção ao longo do tempo, sob hipóteses de exploração ou quando sujeitas a agentes físicos e bióticos (Tomé, 1991; Vanclay, 1994).

No caso das florestas naturais no Brasil, os métodos mais empregados para prognose da produção são a Cadeia de Markov e o método de razão de movimentação (Austregésilo et al., 2004; Teixeira et al., 2007; Stepka et al., 2010), cujas estimativas se restringem ao nível de classes diamétricas ou povoamento. No entanto, torna-se cada vez mais importante o detalhamento das informações acerca do crescimento e dinâmica das florestas em nível de árvore, principalmente para produção de madeira de alta qualidade ou alta importância ecológica e estes métodos não são capazes de oferecer entendimento suficiente em nível de árvore, espécie ou grupo de espécies.

Neste sentido, surge como alternativa o modelo de crescimento em nível de árvore individual (MAI), que embora seja mais complexo, por ser constituído por um conjunto de submodelos, permite ao manejador simular e entender o crescimento das árvores e da floresta sob diferentes condições, como por exemplo, densidade de árvores, competição, entre outros.

Os MAI são compostos por submodelos (Vanclay, 1994), sendo os principais aqueles que estimam a mortalidade e o crescimento das variáveis dendrométricas (altura e diâmetro). Outras variáveis, como dimensões das copas, por exemplo, podem ser consideradas, caso estas sejam variáveis explicativas nos demais submodelos. Além disso, no estudo de florestas naturais, torna-se necessário estimar também o ingresso de novas árvores (Hasenauer, 2000). Com a aplicação destes sub-modelos torna-se possível a simulação do crescimento e produção em nível de árvores individuais.

Assim sendo, este estudo foi desenvolvido com o objetivo de validar um modelo completo em nível de árvore individual utilizando redes neurais artificiais, desenvolvido para um fragmento de Floresta Estacional Semidecidual em estágio médio de sucessão ecológica. 


\section{MATERIAL E MÉTODOS}

\section{Dados}

Os dados utilizados neste estudo foram obtidos no município de Viçosa, Minas Gerais, em um fragmento florestal pertencente à Universidade Federal de Viçosa, que possui 17 ha de área $\left(42^{\circ} 52^{\prime} \mathrm{W}\right.$ e $42^{\circ} 50^{\prime} \mathrm{W}$ de longitude e $20^{\circ} 44^{\prime} \mathrm{S}$ e $20^{\circ} 47^{\prime} \mathrm{S}$ de latitude), pertencente à região fitoecológica de Floresta Estacional Semidecidual Montana em estágio médio de sucessão.

Para o presente trabalho, foram amostrados 0,4 hectares, divididos em quatro parcelas permanentes de $1.000 \mathrm{~m}^{2}(20 \mathrm{~m} \times 50 \mathrm{~m})$. Em cada parcela, foram identificadas as árvores e mensurados os diâmetros a 1,3 m de altura (dap), as alturas total $(H t)$ e do fuste $(H f)$ de todas as árvores com dap $\geq 5 \mathrm{~cm}$, bem como identificados os indivíduos que morreram e ingressaram entre cada medição sucessiva.

Para cada árvore foram determinadas as coordenas $\mathrm{x}$ e y em relação à origem da sua parcela, para criação de um mapa de localização dos indivíduos dentro de cada parcela e estimação dos índices de competição. Os inventários foram realizados nos anos de 1994, 1997, 2000, 2004 e 2008, resultando em um total de 2.062 casos, sendo observados neste período, 181 casos de mortalidade e 146 casos de ingresso. O inventário de 1994 foi utilizado para iniciar a simulação, enquanto os demais foram utilizados apenas para comparação das estimativas do modelo completo aos valores reais.

Todos os indivíduos mensurados foram classificados em relação aos níveis de infestação por cipós, iluminação de copa e qualidade de copa, conforme proposto por Silva \& Lopes (1984), além de serem classificados em grupos ecológicos (Gandolfi et al., 1995) (Tabela 1).

Tabela 1 - Critérios para classificação dos indivíduos em relação à infestação por cipós, iluminação de copa, qualidade da copa e grupo ecológico

\begin{tabular}{|c|c|}
\hline Critérios para classificação & Classe \\
\hline \multicolumn{2}{|l|}{ Infestação por Cipós } \\
\hline Sem presença de cipós & 1 \\
\hline Presença de cipós somente no fuste & 2 \\
\hline Presença de cipós somente na copa & 3 \\
\hline Presença de cipós no fuste e na copa & 4 \\
\hline \multicolumn{2}{|l|}{ Iluminação de copa } \\
\hline Copa recebendo radiação solar direta na sua parte superior e lateral & 1 \\
\hline Copa recebendo radiação solar direta na sua parte superior & 2 \\
\hline Ausência de radiação solar direta na copa & 3 \\
\hline \multicolumn{2}{|l|}{ Qualidade da copa } \\
\hline Boa: copa normal, sem presença de danos & 1 \\
\hline Regular: copa com algum dano de pequena escala & 2 \\
\hline Inferior: copa com severos danos, com poucos galhos e folhas & 3 \\
\hline \multicolumn{2}{|l|}{ Grupo Ecológico } \\
\hline $\begin{array}{l}\text { Espécies dependentes de luz e que não ocorrem no sub- bosque, se } \\
\text { desenvolvendo em clareiras ou bordas da floresta }\end{array}$ & Pioneiras (PI) \\
\hline $\begin{array}{l}\text { Desenvolvem-se em condições de sombreamento médio, como pequenas } \\
\text { clareiras e sub-bosque não densamente sombreado }\end{array}$ & Secundárias iniciais (SI) \\
\hline $\begin{array}{l}\text { Desenvolvem-se no sub-bosque em condições de sombra leve ou densa, } \\
\text { podendo crescer até alcançar o dossel }\end{array}$ & $\begin{array}{l}\text { Secundárias tardias } \\
\text { (ST) }\end{array}$ \\
\hline $\begin{array}{l}\text { Espécies que em função da carência de informações não foram incluídas em } \\
\text { nenhuma das categorias }\end{array}$ & Sem classificação SC) \\
\hline
\end{tabular}

\section{Validação do modelo completo de árvore individual (projeção)}

A validação do modelo completo consistiu em aplicar submodelos de mortalidade, crescimento e ingresso, que interagiram durante a simulação (Figura 1). A projeção durante o intervalo de estudo (1994-2008) foi feita com a utilização apenas dos dados do inventário 
realizado no ano de 1994 das parcelas que não estavam presentes no treinamento das redes (submodelos).

As projeções foram realizadas para os seguintes intervalos de tempo: 1994-1997; 1997-2000; 2000-2004; 2004-2008, e comparadas aos valores observados após a simulação.

\section{Índices de competição}

Para cada fuste (unidade básica do estudo) durante os períodos da simulação, foram calculados um índice de competição dependente da distância para a estimativa de probabilidade de mortalidade $\left(\mathrm{IDD}_{m}\right)$ e dois índices de competição semi-independentes da distância (ISD), para as estimativas do crescimento em diâmetro (ISD $\left.D_{d}\right)$ e crescimento em altura $\left(\mathrm{ISD}_{h}\right)$, cujos estimadores são:

$$
\begin{aligned}
& \operatorname{IDD}_{m}=\sum_{j=1}^{n j} \frac{D A P_{i}}{D^{\prime} P_{j} \cdot L_{i j}} \\
& \operatorname{ISD}_{d}=\frac{\mathrm{Ht}_{i}}{\overline{\mathrm{Ht}}_{\mathrm{n}}} \\
& \mathrm{ISD}_{\mathrm{h}}=\frac{\mathrm{AS}_{\mathrm{i}}{ }^{2}}{\mathrm{ASq}^{2}}
\end{aligned}
$$

Em que: dap $_{\mathrm{i}}=$ diâmetro com casca (dap) do fuste da árvore-objeto medido a $1,30 \mathrm{~m}(\mathrm{~cm}) ; d a p_{\mathrm{j}}=$ diâmetro com casca $(d a p)$ do fuste da árvore-competidora medido a $1,30 \mathrm{~m}(\mathrm{~cm}) ; L_{i j}=$ distância entre a árvore-objeto e a árvore competidora $(\mathrm{cm})$; $n_{j}=$ número de fustes competidores limitados a um raio de competição de $6 \mathrm{~m}$; $H t_{\mathrm{i}}=$ altura total do fuste da árvore-objeto $(\mathrm{m}) ; \overline{\mathrm{Ht}}_{\mathrm{n}}=$ altura média dos fustes de $n$ árvores próximas da árvore-objeto $(\mathrm{m})$, limitadas a um raio de competição de $6 \mathrm{~m}$; $A S_{i}=$ área seccional do fuste da árvore-objeto, medido a $1,30 \mathrm{~m}\left(\mathrm{~m}^{2}\right), A S q=$ área seccional correspondente ao diâmetro médio $(q)$ dos fustes das árvores vizinhas $\left(\mathrm{m}^{2}\right)$.

\section{Submodelo de mortalidade}

Foi utilizada uma rede neural previamente selecionada para estimativa da probabilidade de mortalidade dos indivíduos. Essa é caracterizada por uma rede do tipo Multilayer Perceptron (MLP), com quatorze neurônios na única camada intermediária e com função de ativação exponencial nas camadas intermediária e de saída, utilizando como uma das variáveis independentes (entrada), um índice de competição dependente da distância (Tabela 2).

Tabela 2 - Características da rede neural selecionada para estimar a probabilidade de mortalidade em

\begin{tabular}{|c|c|c|c|c|c|c|c|}
\hline \multirow{2}{*}{$\begin{array}{l}\text { Tipo } \\
\text { de } \\
\text { rede }\end{array}$} & \multirow{2}{*}{$\begin{array}{c}\text { Arquitetura da } \\
\text { Rede para } \\
\text { mortalidade* }\end{array}$} & \multirow{2}{*}{$\begin{array}{c}\text { Saída } \\
\text { (numérica) }\end{array}$} & \multirow{2}{*}{$\begin{array}{l}\text { Entradas } \\
\text { Numéricas }\end{array}$} & \multirow{2}{*}{$\begin{array}{l}\text { Entradas } \\
\text { Categóricas }\end{array}$} & \multicolumn{2}{|c|}{ Função de ativação } & \multirow[b]{2}{*}{$\mathbf{r}_{\hat{y} \mathbf{y}}$} \\
\hline & & & & & $\begin{array}{c}\text { Camada } \\
\text { intermediária }\end{array}$ & $\begin{array}{l}\text { Camada } \\
\text { de saída }\end{array}$ & \\
\hline$P$ & $60-14-1$ & $P m$ & $\begin{array}{c}\mathrm{A}_{1}, \mathrm{~A}_{2}, \operatorname{dap}_{1}, \\
H t_{1}, \mathrm{IDD}_{\mathrm{m}}\end{array}$ & $\begin{array}{c}\mathrm{F}, \mathrm{GE}, \mathrm{C}, \mathrm{IC} \text {, } \\
\mathrm{QC}\end{array}$ & Exponencial & Exponencial & 0,58 \\
\hline
\end{tabular}
uma Floresta Estacional Semidecidual Montana, em estágio médio de sucessão, localizada em Viçosa Minas Gerais.

Em que MLP é uma rede Multilayer Perceptron; (*) indica o número de neurônios da camada de entrada, intermediária e saída, respectivamente; $P m$ é a probabilidade de mortalidade entre dois períodos de medição; $A_{1}$ e $A_{2}$ são os anos de medição atual e futuro, respectivamente; dap 1 é o diâmetro a $1,3 \mathrm{~m}(\mathrm{~cm})$ atual; $H t_{1}$ é a altura total atual; IDDm é o índice de competição dependente da distância; F é a família botânica do indivíduo; GE é o grupo ecológico; C é o nível de infestação por cipó; IC é o nível de iluminação da copa e QC é o nível de qualidade da copa. 
Estimada a probabilidade de mortalidade $P m$ para cada indivíduo em um dado período, esta foi comparada com um número aleatório $(\mathrm{Pa})$ entre zero e um, segundo Pretzsch et al. (2002). A regra de decisão para a mortalidade no período de projeção foi: se $P m>P a, 0$ indivíduo morre, caso contrário, permanece vivo. Como se trata de um processo aleatório, este procedimento foi repetido 30 vezes, a fim de obter uma tendência média da estimativa de mortalidade para cada período de projeção. A amostra que mais se aproximou do valor médio das 30 repetições em cada período foi aquela selecionada para compor a lista de árvores mortas.

\section{Submodelo de crescimento}

Para a projeção do crescimento em dap, altura total $(H t)$ e altura do fuste $(H f)$ simultaneamente, foi utilizada uma rede neural do tipo Multilayer Perceptron (MLP), com 186 neurônios na única camada intermediária e com função de ativação tangencial nas camadas intermediária e de saída, utilizando dentre as variáveis independentes, dois índices de competição semi-independentes da distância (Tabela 3).

Tabela 3 - Características da rede neural selecionada para projetar o dap, altura total $(H t)$ e altura do fuste $(H f)$ das árvores em uma Floresta Estacional Semidecidual Montana, em estágio médio de sucessão, localizada em Viçosa - Minas Gerais

\begin{tabular}{|c|c|c|c|c|c|c|c|}
\hline $\begin{array}{l}\text { Tipo } \\
\text { de } \\
\text { rede }\end{array}$ & $\begin{array}{l}\text { Arquitetura da } \\
\text { Rede para } \\
\text { crescimento* }\end{array}$ & $\begin{array}{c}\text { Saídas } \\
\text { (numérica) }\end{array}$ & $\begin{array}{l}\text { Entradas } \\
\text { Numéricas }\end{array}$ & $\begin{array}{l}\text { Entradas } \\
\text { Categóricas }\end{array}$ & $\begin{array}{c}\mathbf{r}_{\hat{y} y} \\
(d a p)\end{array}$ & $\begin{array}{c}\mathbf{r}_{\hat{y} y} \\
(H t)\end{array}$ & $\begin{array}{c}\mathbf{r}_{\hat{y} \mathbf{y}} \\
(H f)\end{array}$ \\
\hline MLP & $61-186-3$ & $d a p_{2}, H t_{2}, H f_{2}$ & $\begin{array}{c}\mathrm{A}_{1}, \mathrm{~A}_{2}, \operatorname{dap}_{1}, H t_{1} \\
H f_{1}, \mathrm{ISD}_{\mathrm{d}}, \mathrm{ISD}_{\mathrm{h}}\end{array}$ & $\mathrm{F}, \mathrm{GE}, \mathrm{C}, \mathrm{IC}, \mathrm{QC}$ & 0,9952 & 0,9800 & 0,9801 \\
\hline
\end{tabular}

em que MLP é uma rede Multilayer Perceptron; (*) indica o número de neurônios da camada de entrada, intermediária e saída, respectivamente; $d a p_{1}$ e dap 2 são os diâmetros a 1,3 m (cm) atual e futuro, respectivamente; $H t_{1}$ e $H t_{2}$ são as alturas totais ( $\mathrm{m}$ ) atual e futura, respectivamente; $\mathrm{Hf}_{1}$ e $H f_{2}$ são as alturas dos fustes ( $\mathrm{m}$ ) atual e futura, respectivamente; $A_{1}$ e $A_{2}$ são as medições atuais e futuras, respectivamente; ISDd e ISDh são índices de competição semi-dependentes da distância; F é a família botânica do indivíduo; GE é o grupo ecológico; C é o nível de infestação por cipó; IC é o nível de iluminação da copa e QC é o nível de qualidade da copa.

\section{Submodelo de ingresso}

Devido à ausência de dados coletados de regeneração $($ dap $<5 \mathrm{~cm})$ durante o período de estudo, não existiam informações que antecedessem o evento de ingresso das árvores para o treinamento de redes neurais artificiais. Para que a simulação do crescimento e produção da floresta não fosse comprometida pela falta deste submodelo, foi determinada uma taxa média de ingresso a partir dos mesmos dados que originaram os demais submodelos, e essa taxa foi adotada para cada período de projeção nos dados de validação.

O indivíduo que ingressaria durante o periodo de projeção foi definido de forma aleatória, com base na listagem das árvores observada no período de estudo. Cada indivíduo que ingressou foi alocado em uma das parcelas de validação, também de forma aleatória.

Para que fosse possível o cálculo dos índices de competição dependentes e semiindependentes da distância durante a simulação, foi definido aleatoriamente coordenadas $x$ (entre 0 e $20 \mathrm{~m}$ ) e y (entre 0 e $50 \mathrm{~m}$ ) para que essas árvores que ingressaram se posicionassem nas parcelas.

Foi estipulado também que todos os ingressos possuíssem dap igual a $5,92 \mathrm{~cm}$, altura total igual a 7,42 m e altura do fuste igual a 4,47 m, conforme valores médios das árvores ingressas (observados nos dados para geração dos sub-modelos). As qualificações quanto ao nível de infestação por cipó e o nível de qualidade da copa foram iguais "1" e nível de iluminação da copa igual a "3". Adotou-se também que as árvores ingressas não se bifurcavam durante a simulação. 


\section{Avaliação da projeção aplicando o modelo completo de árvore individual}

O volume de cada indivíduo no ano final da projeção (ano de 2008) foi estimado de acordo com a fórmula do Centro Tecnológico de Minas Gerais (1995), para florestas em estágio sucessional secundário:

$\mathrm{VTcc}=0,000074230 \cdot \mathrm{dap}^{1,707348} \cdot \mathrm{Ht}^{1,16873} \mathrm{R}^{2}=0,972$

em que: VTcc é o volume total com casca, em $\mathrm{m}^{3}$; dap é o diâmetro à 1,3 $\mathrm{m}$, em cm; Ht é a altura total em $m$ e $R^{2}$ é o coeficiente de determinação.

Foram elaborados gráficos de barras e de dispersão para comparar os dados projetados (validação) com os dados observados, em diferentes níveis de detalhamento (povoamento total, distribuição diamétrica e de altura, e árvore individual), considerando as seguintes variáveis:

a) volume por hectare para cada parcela no último ano de medição (2008);

b) frequência de indivíduos para cada parcela no ano de 2008;

c) frequências de indivíduos mortos e ingressos nos quatro períodos de projeção;

d) distribuição diamétrica e de altura no último ano de medição (2008).

Para verificar a aderência dessas estimativas com os valores observados utilizou-se o teste não paramétrico de Kolmogorov-Smirnov (K-S) (Sokal \& Rohlf, 1969), cuja estatística é dada por:

$\operatorname{dn}=\operatorname{Max}\left|F_{o}(x)-F_{e}(x)\right|$

Em que: $d n$ é o valor calculado da estatística de K-S; $F_{0}(X)$ é a frequência acumulada observada; $F_{e}(x)$ é a frequência acumulada estimada.

A hipótese nula ( $H_{0}$ : as estimativas não diferem dos valores reais) foi rejeitada para o valor calculado de $d n$ maior que o valor tabelado, ao nível de significância a igual a 5\%.

Para avaliar a precisão das projeções do dap, altura total e altura do fuste, foram elaborados gráficos de dispersão, histogramas de resíduos, bem como calculadas as estatísticasde correlação $\left(\mathrm{r}_{\mathrm{y} y \mathrm{y}}\right)$ e a raiz do erro quadrado médio (RMSE) entre os dados projetados e observados no ano de 2008. Quanto maior o coeficiente de correlação (próximo a 1) e menor a RMSE, melhor a previsão da estimativa.

$r_{\hat{y} y}=\frac{\operatorname{cov}(Y, \hat{Y})}{\sqrt{S^{2}(Y) S^{2}(\hat{Y})}}$

$\operatorname{RMSE}(\%)=100 \cdot \sqrt{\frac{\sum_{i=1}^{n}(Y i-\hat{Y} i)^{2}}{n}} / \bar{Y}$

Em que: $Y_{i}$ é a variável de saída observada; $\hat{Y}_{i}$ é a variável de saída estimada; cov é a covariância; $S^{2}$ é a variância; $n$ é o número total de dados; $\bar{Y}$ é a média da saída observada.

Realizaram-se também comparações entre os índices de valor de importância (IVI\%) (Soares et al., 2011) da estrutura horizontal observada e simulada para o ano de 2008, pelo teste F (Graybill, 1976). 


\section{RESULTADOS E DISCUSSÃO}

Pela totalização dos volumes das árvores em cada parcela de validação no ano de 2008, ano final da simulação, observou-se leve tendência de subestimação dos volumes por hectare em torno de $5 \%$ (Figura 2), diferença que não foi significativa pelo teste $\mathrm{K}-\mathrm{S}(p<0,05)$. Devido à variabilidade de produtividade entre as parcelas na área de estudo (volumes entre 245 e $637 \mathrm{~m}^{3} \mathrm{ha}^{-1}$ ), em função principalmente da localização das parcelas na área e presença de árvores remanescentes de grande porte, a diferença entre os valores observados e as estimativas pode ser considerada satisfatória.

Em relação à frequência dos indivíduos estimados para o ano de 2008, observa-se precisão das estimativas - teste K-S não siginficativo $(p<0,05)$, com desvio médio de $0,3 \%$ (Figura 2b).

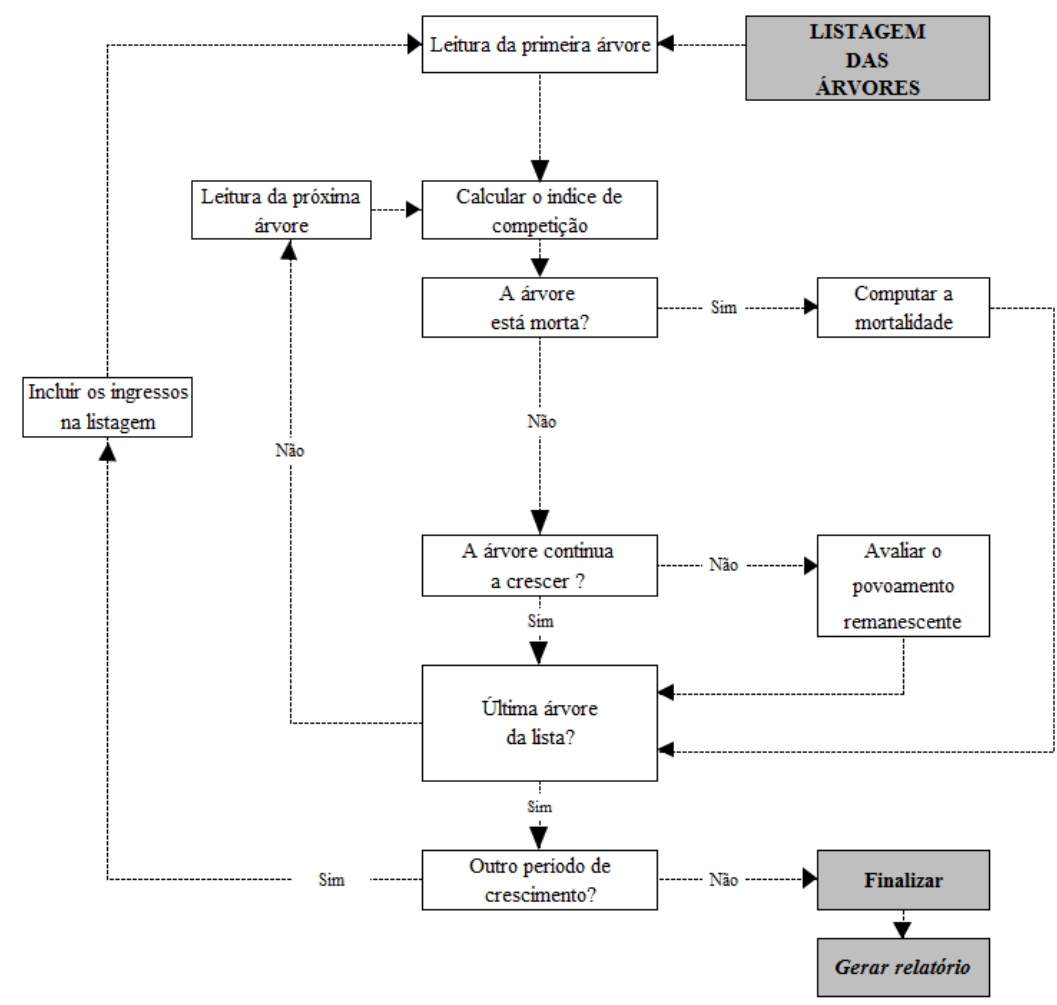

Figura 1 - Fluxograma para utilização de um modelo em nível de árvore individual em uma floresta natural. Fonte: Adaptado de Davis \& Johnson (1987).

Pela análise da Figura 3a pode-se inferir que o sub-modelo de mortalidade foi eficiente durante o período de simulação, sendo observado um desvio médio de -7\%. A precisão dessa estimativa foi confirmada pelo teste K-S $(p<0,05)$.

A tendência obtida na validação do modelo está em conformidade com resultados descritos na literatura para estimativas de mortalidade (Monserud \& Sterba, 1999; Taylor \& Maclean, 2007). Uma característica importante para a modelagem de mortalidade é a necessidade de um banco de dados que represente bem a população em estudo (Monserud \& Sterba, 1999). No presente trabalho, apesar do grande número de árvores, o número de árvores mortas não foi tão grande, e mesmo assim as estimativas obtidas para mortalidade foram precisas.

Em relação à frequência de árvores ingressas durante os períodos de projeção (Figura 3b), foi observado um desvio médio de $-22 \%$ em relação aos valores observados, e apresentou estimativas estatisticamente diferentes dos dados reais para essa variável pelo teste K-S $(p>0,05)$. Constatou-se que o ponto de maior divergência para 0 ingresso foi no último intervalo de projeção, o que ocasionou a maior diferença. 
Em outros trabalhos, o ingresso também foi tratado como problema em simulações do crescimento e produção em nível de distribuição diamétrica (Pulz, 1998; Austregésilo et al., 2004).

(a)

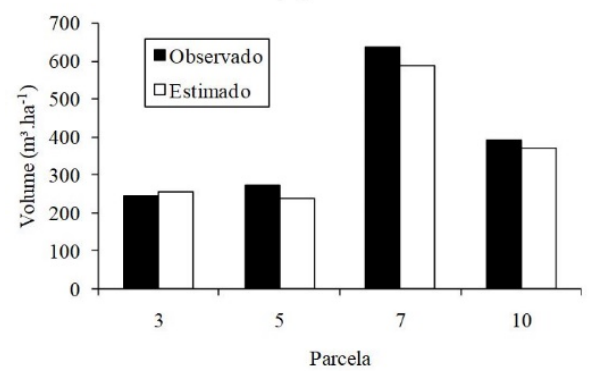

(b)

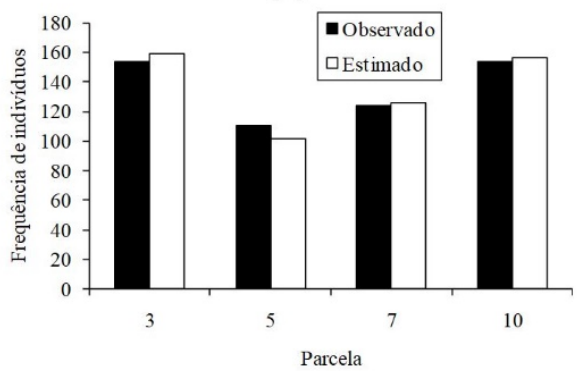

Figura 2 - Volume por hectare observado e estimado pelo modelo completo em nível de árvore individual (Teste K-S: 0,05 n.s (a) e frequência de indivíduos observados e estimados (Teste K-S: 0,09n.s) (b) para as parcelas de validação, no ano de 2008.

O submodelo de ingresso deve ser aprimorado em estudos de crescimento e produção em nível de árvore individual para florestas naturais. Para tanto, torna-se necessária a coleta de informações que antecedem esse evento (dados de regeneração), como variáveis explicativas para realização da previsão com maior eficiência.

As estimativas da frequência de indivíduos por classe de diâmetro e por classe de altura (Figuras $4 a, 4 b$ ) foram estatisticamente iguais aos valores observados no ano de 2008 pelo teste K-S $(p<0,05)$.

Cabe-se destacar que este é o nível de detalhamento obtido pelos principais trabalhos que simulam o crescimento de florestas naturais no Brasil (nível de distribuição diamétrica pelo método da matriz de transição - Cadeia de Markov) (Freitas \& Higuchi, 1993; Azevedo et al., 1995; Sanquetta et al., 1996; Pulz et al., 1999; Arce et al., 2001; Austregésilo et al., 2004; Menon et al., 2010; Stepka et al., 2010). Na maioria destes trabalhos, apesar de obtido sucesso na modelagem, foi identificada falta de consistência nas menores classes de diâmetro, principalmente devido ao ingresso, o que não se verificou neste trabalho com a simulação (Figura 4).

(a)

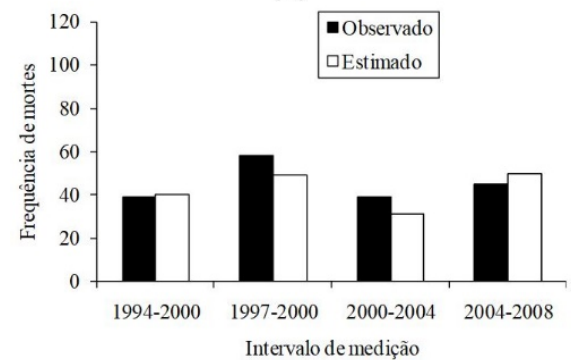

(b)

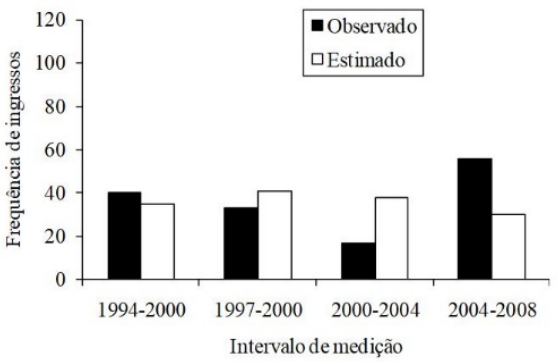

Figura 3 - Frequência de indivíduos mortos (Teste K-S: 0,074 ${ }^{\text {n.s }}$ ) (a) e ingressos (Teste K-S: 0,168*) (b), observados e estimados pelo modelo completo em nível de árvore individual no período de projeção (1994-2008).

Em nível de árvore individual, observa-se, nas Figuras $5 a$, 5b e $5 c$, a exatidão das estimativas do modelo completo para estimativa das variáveis dap (a), altura total (b) e altura do fuste (c), haja vista a concentração das estimativas dos erros em torno de zero e tendência de distribuição das estimativas e dos valores observados em torno da linha de $45^{\circ}$ no gráfico de dispersão. 
(a)

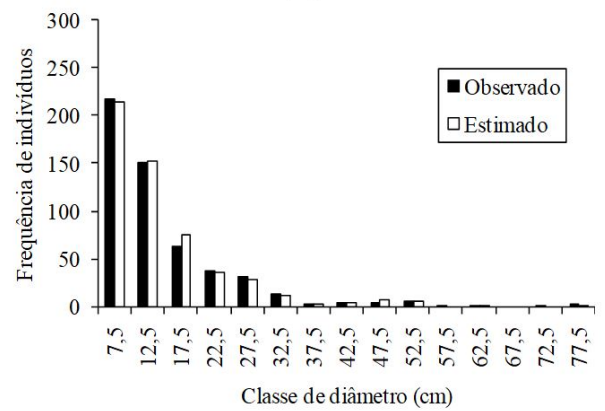

(b)

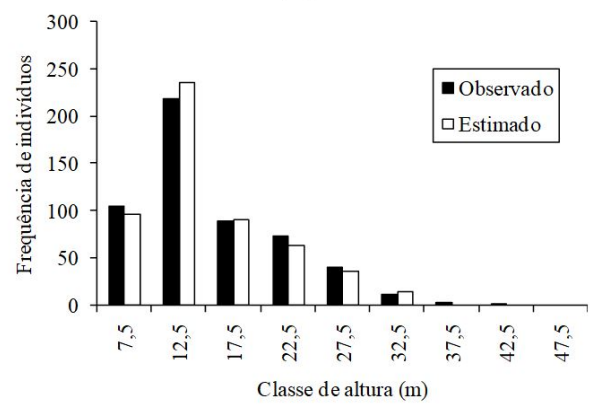

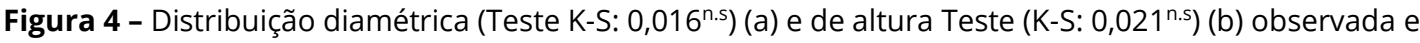
estimada pelo modelo de árvore individual no ano final de projeção (2008).

(a)
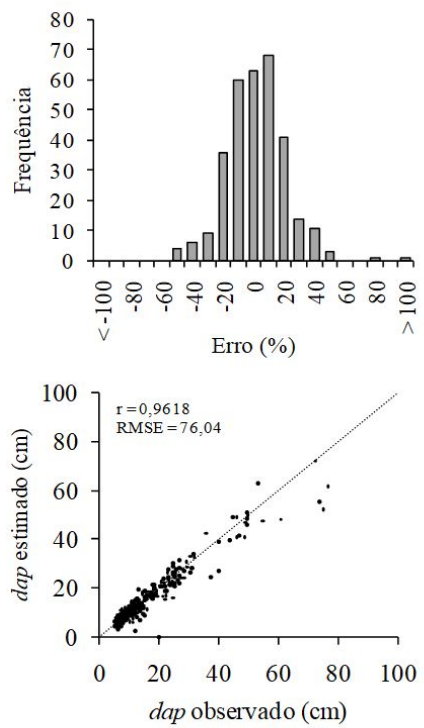

(b)
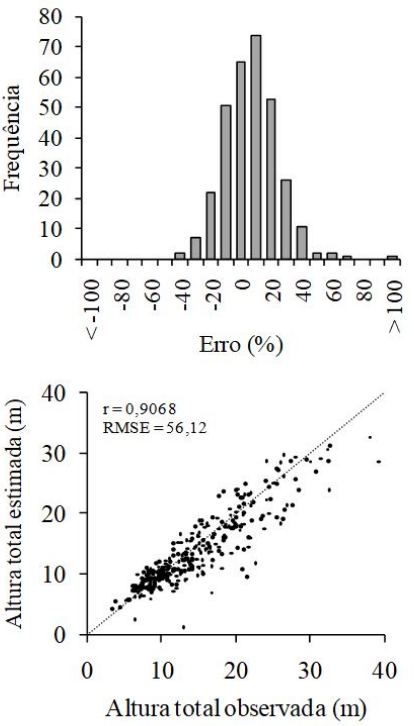

(c)
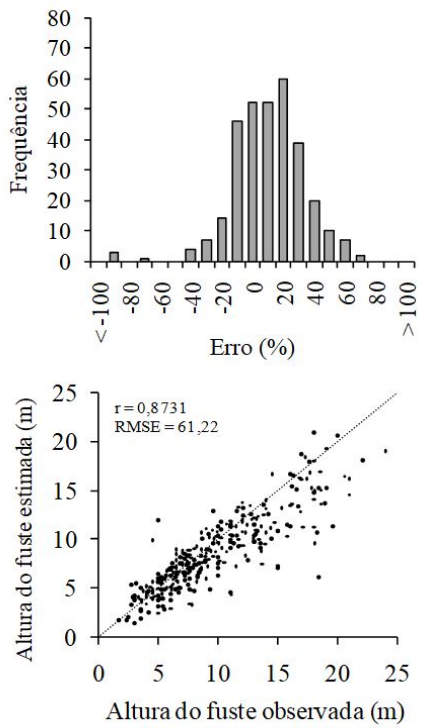

Figura 5 - Histograma de resíduos, e valores observados versus estimados de dap (a), altura total (b) e altura do fuste (c) obtidas pelo modelo completo em nível de árvore individual no ano final de projeção (2008).

Este modelo em nível de árvore individual apresenta algumas vantagens em relação aos tradicionais modelos de prognose de florestas naturais, os quais consideram apenas dois períodos de medição para construírem os modelos com base em uma matriz de probabilidade. Neste trabalho, as projeções não contemplam apenas as alterações obtidas em um único período, mas alterações de quatro períodos (1994-1997; 1997-2000; 2000-2004; 2004-2008). Outra vantagem que merece destaque é que o período de projeção não é limitado a apenas períodos múltiplos como àqueles que originaram o modelo.

Ao comparar a estrutura horizontal (EH) resultante da simulação no ano de $2008 \mathrm{com}$ a observada, verificou-se que das 17 espécies que representam juntas mais de 50\% do IVI da floresta na EH observada, 14 delas (82\%) também se apresentaram entre as 17 mais importantes na estrutura projetada.

Pela comparação do IVI das espécies das estruturas observada e estimada, verificou coincidência pelo teste $F$, indicando que a simulação foi precisa e não influenciou significativamente a estrutura horizontal da floresta no ano final da simulação $(p>0,05$; $\mathrm{R}^{2}=92,85 \% ; \overline{\mathrm{E}}=4,9 \%$ ) (Tabela 4). 
Tabela 4 - Estrutura horizontal das parcelas de validação observada e estimada pelo modelo completo de árvore individual no de 2008, em uma Floresta Estacional Semidecidual em estágio médio de sucessão ecológica.

\begin{tabular}{|c|c|c|c|c|c|c|c|c|c|c|}
\hline \multirow[b]{2}{*}{ Nome Científico } & \multicolumn{5}{|c|}{ Estrutura Horizontal Observada } & \multicolumn{5}{|c|}{ Estrutura Horizontal Estimada } \\
\hline & DR & DoR & $\mathbf{F R}$ & IVI & $\begin{array}{c}\text { Ranking } \\
\text { IVI }\end{array}$ & DR & DoR & FR & IVI & $\begin{array}{c}\text { Ranking } \\
\text { IVI }\end{array}$ \\
\hline Anadenanthera peregrina & 4,24 & 21,04 & 2,25 & 9,18 & 1 & 5,25 & 20,68 & 2,05 & 9,33 & 1 \\
\hline Newtonia contorta & 5,71 & 18,46 & 1,69 & 8,62 & 2 & 3,89 & 13,11 & 1,03 & 6,01 & 2 \\
\hline Bathysa nicholsonii & 10,87 & 2,98 & 1,69 & 5,18 & 3 & 8,95 & 2,52 & 2,05 & 4,51 & 4 \\
\hline Siparuna arianeae & 10,87 & 1,58 & 2,25 & 4,90 & 4 & 11,67 & 2,20 & 2,05 & 5,31 & 3 \\
\hline Casearia ulmifolia & 4,05 & 4,01 & 2,25 & 3,44 & 5 & 3,11 & 2,92 & 2,05 & 2,69 & 5 \\
\hline Piptadenia gonoacantha & 3,13 & 2,58 & 1,12 & 2,28 & 6 & 2,92 & 3,06 & 1,03 & 2,33 & 7 \\
\hline Apuleia leiocarpa & 2,21 & 2,09 & 1,69 & 1,99 & 7 & 1,56 & 1,25 & 1,54 & 1,45 & 16 \\
\hline Aparisthmium cordatum & 2,95 & 0,66 & 1,69 & 1,76 & 8 & 4,47 & 1,13 & 2,05 & 2,55 & 6 \\
\hline Machaerium floridum & 1,66 & 1,73 & 1,69 & 1,69 & 9 & 2,14 & 2,62 & 1,54 & 2,10 & 8 \\
\hline Platypodium elegans & 1,10 & 2,83 & 1,12 & 1,69 & 10 & 1,17 & 3,35 & 1,03 & 1,85 & 10 \\
\hline Albizia polycephala & 1,47 & 2,45 & 1,12 & 1,68 & 11 & 1,75 & 3,18 & 1,03 & 1,99 & 9 \\
\hline Cecropia hololeuca & 0,74 & 2,99 & 1,12 & 1,62 & 12 & 0,58 & 2,76 & 1,03 & 1,46 & 15 \\
\hline Casearia arborea & 1,10 & 2,40 & 0,56 & 1,36 & 13 & 0,97 & 2,15 & 0,51 & 1,21 & 21 \\
\hline Rollinia sylvatica & 1,29 & 1,63 & 1,12 & 1,35 & 14 & 2,14 & 2,13 & 1,03 & 1,77 & 11 \\
\hline Allophylus edulis & 1,47 & 0,81 & 1,69 & 1,32 & 15 & 0,97 & 0,54 & 1,54 & 1,02 & 26 \\
\hline Lacistema pubescens & 1,47 & 0,69 & 1,69 & 1,28 & 16 & 2,14 & 0,92 & 1,54 & 1,53 & 13 \\
\hline Myrcia formosiana & 1,47 & 0,68 & 1,69 & 1,28 & 17 & 1,36 & 0,79 & 1,54 & 1,23 & 20 \\
\hline Prunus sellowii & 2,03 & 0,66 & 1,12 & 1,27 & 18 & 1,56 & 0,51 & 1,03 & 1,03 & 24 \\
\hline Trichilia lepidota & 1,47 & 0,59 & 1,69 & 1,25 & 19 & 1,17 & 0,74 & 1,03 & 0,98 & 28 \\
\hline Trichilia catigua & 1,47 & 0,58 & 1,69 & 1,25 & 20 & 1,56 & 0,52 & 2,05 & 1,37 & 17 \\
\hline Ocotea pubescens & 1,66 & 0,32 & 1,69 & 1,22 & 21 & 0,97 & 0,25 & 1,54 & 0,92 & 32 \\
\hline Ocotea odorifera & 1,47 & 0,41 & 1,69 & 1,19 & 22 & 1,36 & 0,42 & 1,03 & 0,94 & 31 \\
\hline Heisteria silviani & 1,10 & 1,20 & 1,12 & 1,14 & 23 & 0,78 & 1,11 & 1,03 & 0,97 & 30 \\
\hline Nectandra lanceolata & 0,55 & 2,24 & 0,56 & 1,12 & 24 & 0,58 & 1,91 & 0,51 & 1,00 & 27 \\
\hline Luehea speciosa & 0,74 & 0,34 & 2,25 & 1,11 & 25 & 0,97 & 1,87 & 1,54 & 1,46 & 14 \\
\hline Rapanea umbellata & 0,92 & 0,55 & 1,69 & 1,05 & 26 & 0,78 & 0,58 & 1,03 & 0,80 & 37 \\
\hline Ocotea dispersa & 0,92 & 0,40 & 1,69 & 1,00 & 27 & 1,17 & 0,55 & 2,05 & 1,26 & 19 \\
\hline Jacaranda macrantha & 1,29 & 0,44 & 1,12 & 0,95 & 28 & 1,75 & 0,69 & 1,54 & 1,33 & 18 \\
\hline Tovomitopsis saldanhae & 1,29 & 0,86 & 0,56 & 0,90 & 29 & 1,36 & 1,22 & 0,51 & 1,03 & 23 \\
\hline Rollinia laurifolia & 0,55 & 0,91 & 1,12 & 0,86 & 30 & 0,39 & 0,28 & 1,03 & 0,56 & 48 \\
\hline Amaioua guianensis & 0,55 & 0,23 & 1,69 & 0,82 & 31 & 0,39 & 0,21 & 1,03 & 0,54 & 50 \\
\hline Endlicheria paniculata & 0,92 & 0,42 & 1,12 & 0,82 & 32 & 0,97 & 0,83 & 1,54 & 1,11 & 22 \\
\hline Inga Alba & 0,55 & 1,32 & 0,56 & 0,81 & 33 & 0,39 & 1,13 & 0,51 & 0,68 & 43 \\
\hline Machaerium stipitatum & 0,74 & 0,50 & 1,12 & 0,79 & 34 & 0,58 & 0,61 & 1,03 & 0,74 & 40 \\
\hline Vitex sellowiana & 0,37 & 1,39 & 0,56 & 0,77 & 35 & 0,19 & 0,23 & 0,51 & 0,31 & 79 \\
\hline Trichilia pallida & 0,74 & 0,43 & 1,12 & 0,76 & 36 & 0,39 & 0,16 & 1,03 & 0,52 & 52 \\
\hline Brosimum sellowii & 0,55 & 0,52 & 1,12 & 0,73 & 37 & 0,39 & 0,15 & 0,51 & 0,35 & 71 \\
\hline Machaerium brasiliense & 0,37 & 1,17 & 0,56 & 0,70 & 38 & 0,19 & 0,59 & 0,51 & 0,43 & 65 \\
\hline Sorocea bonplandii & 0,74 & 0,18 & 1,12 & 0,68 & 39 & 0,39 & 0,07 & 1,03 & 0,50 & 57 \\
\hline Casearia decandra & 0,37 & 0,55 & 1,12 & 0,68 & 40 & 0,58 & 0,70 & 1,03 & 0,77 & 38 \\
\hline Mabea fistullifera & 0,92 & 0,49 & 0,56 & 0,66 & 41 & 1,95 & 0,92 & 2,05 & 1,64 & 12 \\
\hline Copaifera langsdorffii & 0,74 & 0,62 & 0,56 & 0,64 & 42 & 0,58 & 0,55 & 1,03 & 0,72 & 41 \\
\hline Sapium glandulatum & 0,37 & 0,99 & 0,56 & 0,64 & 43 & 0,39 & 1,52 & 0,51 & 0,81 & 36 \\
\hline Ocotea minarum & 0,37 & 0,97 & 0,56 & 0,63 & 44 & 0,19 & 0,43 & 0,51 & 0,38 & 69 \\
\hline Guettarda viburnoides & 0,55 & 0,18 & 1,12 & 0,62 & 45 & 0,19 & 0,14 & 0,51 & 0,28 & 83 \\
\hline Myrcia anceps & 0,55 & 0,17 & 1,12 & 0,62 & 46 & 0,19 & 0,02 & 0,51 & 0,24 & 113 \\
\hline Citrus sp. & 0,92 & 0,34 & 0,56 & 0,61 & 47 & 0,39 & 0,05 & 1,03 & 0,49 & 59 \\
\hline Casearia sylvestris & 0,92 & 0,34 & 0,56 & 0,61 & 48 & 1,56 & 0,49 & 1,03 & 1,02 & 25 \\
\hline Erythroxylum pelleterianum & 0,55 & 0,10 & 1,12 & 0,59 & 49 & 0,58 & 0,24 & 1,03 & 0,62 & 44 \\
\hline
\end{tabular}


Tabela 4 - Continuação...

\begin{tabular}{|c|c|c|c|c|c|c|c|c|c|c|}
\hline \multirow[b]{2}{*}{ Nome Científico } & \multicolumn{5}{|c|}{ Estrutura Horizontal Observada } & \multicolumn{5}{|c|}{ Estrutura Horizontal Estimada } \\
\hline & DR & DoR & FR & IVI & $\begin{array}{c}\text { Ranking } \\
\text { IVI }\end{array}$ & DR & DoR & $\mathbf{F R}$ & IVI & $\begin{array}{c}\text { Ranking } \\
\text { IVI }\end{array}$ \\
\hline Ocotea corymbosa & 0,55 & 0,09 & 1,12 & 0,59 & 50 & 0,58 & 0,13 & 1,03 & 0,58 & 46 \\
\hline Inga affinis & 0,37 & 0,77 & 0,56 & 0,57 & 51 & 0,39 & 0,72 & 0,51 & 0,54 & 51 \\
\hline Platymiscium pubescens & 0,55 & 0,57 & 0,56 & 0,56 & 52 & 0,97 & 1,06 & 0,51 & 0,85 & 34 \\
\hline Eugenia florida & 0,37 & 0,16 & 1,12 & 0,55 & 53 & 0,19 & 0,05 & 0,51 & 0,25 & 92 \\
\hline Virola sebifera & 0,18 & 0,88 & 0,56 & 0,54 & 54 & - & - & - & - & - \\
\hline Dalbergia nigra & 0,37 & 0,67 & 0,56 & 0,53 & 55 & 0,39 & 2,03 & 0,51 & 0,98 & 29 \\
\hline Guatteria villosissima & 0,37 & 0,10 & 1,12 & 0,53 & 56 & 0,39 & 0,10 & 1,03 & 0,51 & 53 \\
\hline Cabralea canjerana & 0,37 & 0,09 & 1,12 & 0,53 & 57 & - & - & - & - & - \\
\hline Carpotroche brasiliensis & 0,37 & 0,07 & 1,12 & 0,52 & 58 & 0,19 & 0,04 & 0,51 & 0,25 & 97 \\
\hline Guapira opposita & 0,37 & 0,07 & 1,12 & 0,52 & 59 & 0,39 & 0,14 & 0,51 & 0,35 & 73 \\
\hline Maytenus robusta & 0,55 & 0,40 & 0,56 & 0,50 & 60 & 0,39 & 0,60 & 0,51 & 0,50 & 56 \\
\hline Tapirira peckoltiana & 0,55 & 0,20 & 0,56 & 0,44 & 61 & 0,58 & 0,23 & 0,51 & 0,44 & 64 \\
\hline Melanoxylon brauna & 0,55 & 0,16 & 0,56 & 0,42 & 62 & 0,39 & 0,16 & 0,51 & 0,35 & 70 \\
\hline Sloanea monosperma & 0,37 & 0,30 & 0,56 & 0,41 & 63 & 0,39 & 0,27 & 0,51 & 0,39 & 68 \\
\hline Guatteria australis & 0,18 & 0,45 & 0,56 & 0,40 & 64 & 0,39 & 0,43 & 1,03 & 0,61 & 45 \\
\hline Hymatanthus phagedaenicus & 0,37 & 0,25 & 0,56 & 0,39 & 65 & 0,39 & 0,06 & 0,51 & 0,32 & 77 \\
\hline Palicourea guianensis & 0,37 & 0,25 & 0,56 & 0,39 & 66 & 0,19 & 0,33 & 0,51 & 0,35 & 74 \\
\hline Qualea jundiahy & 0,18 & 0,43 & 0,56 & 0,39 & 67 & 0,19 & 0,34 & 0,51 & 0,35 & 72 \\
\hline Tibouchina granulosa & 0,18 & 0,41 & 0,56 & 0,38 & 68 & 0,19 & 0,65 & 0,51 & 0,45 & 61 \\
\hline Hieronyma alchorneoides & 0,18 & 0,40 & 0,56 & 0,38 & 69 & 0,19 & 0,50 & 0,51 & 0,40 & 66 \\
\hline Psychotria carthagenensis & 0,37 & 0,17 & 0,56 & 0,37 & 70 & 0,19 & 0,07 & 0,51 & 0,26 & 91 \\
\hline Alchornea triplinervia & 0,18 & 0,32 & 0,56 & 0,36 & 71 & 0,19 & 0,31 & 0,51 & 0,34 & 75 \\
\hline Siparuna reginae & 0,18 & 0,32 & 0,56 & 0,35 & 72 & 0,19 & 0,25 & 0,51 & 0,32 & 78 \\
\hline Guatteria nigrescens & 0,37 & 0,13 & 0,56 & 0,35 & 73 & 0,19 & 0,03 & 0,51 & 0,25 & 110 \\
\hline Lamanonia ternata & 0,37 & 0,12 & 0,56 & 0,35 & 74 & 0,19 & 0,14 & 0,51 & 0,28 & 84 \\
\hline Alchornea iricurana & 0,37 & 0,09 & 0,56 & 0,34 & 75 & 0,78 & 0,37 & 1,54 & 0,90 & 33 \\
\hline Tabernaemontana fuchsiaefolia & 0,18 & 0,26 & 0,56 & 0,34 & 76 & 0,19 & 0,00 & 0,51 & 0,24 & 118 \\
\hline Rheedia gardneriana & 0,37 & 0,07 & 0,56 & 0,33 & 77 & - & - & - & - & - \\
\hline Psychotria axilaris & 0,37 & 0,05 & 0,56 & 0,33 & 78 & 0,19 & 0,04 & 0,51 & 0,25 & 94 \\
\hline Guarea pendula & 0,37 & 0,05 & 0,56 & 0,33 & 79 & 0,39 & 0,09 & 1,03 & 0,50 & 54 \\
\hline Eugenia leptoclada & 0,37 & 0,05 & 0,56 & 0,33 & 80 & 0,78 & 0,12 & 1,54 & 0,81 & 35 \\
\hline Myrciaria sp. & 0,37 & 0,04 & 0,56 & 0,32 & 81 & 0,19 & 0,03 & 0,51 & 0,25 & 107 \\
\hline Miconia candolleana & 0,18 & 0,21 & 0,56 & 0,32 & 82 & 0,19 & 0,18 & 0,51 & 0,30 & 80 \\
\hline Vernonia diffusa & 0,18 & 0,16 & 0,56 & 0,30 & 83 & 0,39 & 0,45 & 0,51 & 0,45 & 62 \\
\hline Lonchocarpus muehlbergianus & 0,18 & 0,13 & 0,56 & 0,29 & 84 & - & - & - & - & - \\
\hline Mollinedia micrantha & 0,18 & 0,12 & 0,56 & 0,29 & 85 & 0,19 & 0,03 & 0,51 & 0,25 & 101 \\
\hline Cariniana estrellensis & 0,18 & 0,12 & 0,56 & 0,29 & 86 & - & - & - & - & - \\
\hline Matayba elaeagnoides & 0,18 & 0,10 & 0,56 & 0,28 & 87 & 0,39 & 0,63 & 1,03 & 0,68 & 42 \\
\hline Stryphnodendron guianensis & 0,18 & 0,09 & 0,56 & 0,28 & 88 & 0,19 & 0,09 & 0,51 & 0,26 & 86 \\
\hline Hortia arborea & 0,18 & 0,08 & 0,56 & 0,27 & 89 & 0,39 & 0,72 & 0,51 & 0,54 & 49 \\
\hline Bauhinia forficata & 0,18 & 0,07 & 0,56 & 0,27 & 90 & 0,19 & 0,07 & 0,51 & 0,26 & 90 \\
\hline Xylosma sp. & 0,18 & 0,04 & 0,56 & 0,26 & 91 & 0,39 & 0,29 & 0,51 & 0,40 & 67 \\
\hline Sparattosperma leucanthum & 0,18 & 0,04 & 0,56 & 0,26 & 92 & 0,19 & 0,03 & 0,51 & 0,25 & 111 \\
\hline Trichilia claussennii & 0,18 & 0,03 & 0,56 & 0,26 & 93 & 0,19 & 0,04 & 0,51 & 0,25 & 96 \\
\hline Prockia sp. & 0,18 & 0,03 & 0,56 & 0,26 & 94 & - & - & - & - & - \\
\hline Indeterminada 27 & 0,18 & 0,03 & 0,56 & 0,26 & 95 & 0,39 & 0,01 & 1,03 & 0,48 & 60 \\
\hline Phyllostemonodaphne geminiflora & 0,18 & 0,03 & 0,56 & 0,26 & 96 & - & - & - & - & - \\
\hline Ceiba speciosa & 0,18 & 0,03 & 0,56 & 0,26 & 97 & - & - & - & - & - \\
\hline Indeterminada 01 & 0,18 & 0,03 & 0,56 & 0,26 & 98 & 0,19 & 0,01 & 0,51 & 0,24 & 117 \\
\hline Swartzia elegans & 0,18 & 0,02 & 0,56 & 0,26 & 99 & 0,19 & 0,01 & 0,51 & 0,24 & 115 \\
\hline Zanthoxylum riedelianum & 0,18 & 0,02 & 0,56 & 0,26 & 100 & 0,19 & 0,01 & 0,51 & 0,24 & 116 \\
\hline Miconia sellowiana & 0,18 & 0,02 & 0,56 & 0,26 & 101 & - & - & - & - & - \\
\hline Siparuna guianensis & 0,18 & 0,02 & 0,56 & 0,26 & 102 & 0,19 & 0,03 & 0,51 & 0,25 & 106 \\
\hline
\end{tabular}


Tabela 4 - Continuação...

\begin{tabular}{|c|c|c|c|c|c|c|c|c|c|c|}
\hline \multirow[b]{2}{*}{ Nome Científico } & \multicolumn{5}{|c|}{ Estrutura Horizontal Observada } & \multicolumn{5}{|c|}{ Estrutura Horizontal Estimada } \\
\hline & DR & DoR & FR & IVI & $\begin{array}{c}\text { Ranking } \\
\text { IVI }\end{array}$ & DR & DoR & FR & IVI & $\begin{array}{c}\text { Ranking } \\
\text { IVI }\end{array}$ \\
\hline Indeterminada 33 & 0,18 & 0,02 & 0,56 & 0,26 & 103 & - & - & - & - & - \\
\hline Maytenus aquifolium & 0,18 & 0,02 & 0,56 & 0,26 & 104 & 0,39 & 0,08 & 1,03 & 0,50 & 55 \\
\hline Anadenanthera colubrina & 0,18 & 0,02 & 0,56 & 0,26 & 105 & - & - & - & - & - \\
\hline Nectandra cf. saligna & 0,18 & 0,02 & 0,56 & 0,26 & 106 & - & - & - & - & - \\
\hline Piper arboreum & 0,18 & 0,02 & 0,56 & 0,25 & 107 & 0,19 & 0,14 & 0,51 & 0,28 & 85 \\
\hline Maprounea guianensis & 0,18 & 0,02 & 0,56 & 0,25 & 108 & - & - & - & - & - \\
\hline Coussarea verticilata & 0,18 & 0,02 & 0,56 & 0,25 & 109 & 0,19 & 0,04 & 0,51 & 0,25 & 95 \\
\hline Andira fraxinifolia & 0,18 & 0,02 & 0,56 & 0,25 & 110 & 0,19 & 0,03 & 0,51 & 0,25 & 108 \\
\hline Psychotria sp. & 0,18 & 0,02 & 0,56 & 0,25 & 111 & 0,19 & 0,04 & 0,51 & 0,25 & 98 \\
\hline Lauraceae 3 & 0,18 & 0,02 & 0,56 & 0,25 & 112 & 0,19 & 0,04 & 0,51 & 0,25 & 99 \\
\hline Miconia sp. & - & - & - & - & - & 0,58 & 0,12 & 1,54 & 0,75 & 39 \\
\hline Croton urucurana & - & - & - & - & - & 0,19 & 0,99 & 0,51 & 0,57 & 47 \\
\hline Myrcia sp. & - & - & - & - & - & 0,39 & 0,07 & 1,03 & 0,49 & 58 \\
\hline Cassia ferruginea & - & - & - & - & - & 0,19 & 0,63 & 0,51 & 0,44 & 63 \\
\hline Psychotria sessilis & - & - & - & - & - & 0,39 & 0,08 & 0,51 & 0,33 & 76 \\
\hline Solanum leucodendron & - & - & - & - & - & 0,19 & 0,16 & 0,51 & 0,29 & 81 \\
\hline Indeterminada 15 & - & - & - & - & - & 0,19 & 0,15 & 0,51 & 0,28 & 82 \\
\hline Acacia polyphila & - & - & - & - & - & 0,19 & 0,08 & 0,51 & 0,26 & 87 \\
\hline Nectandra rigida & - & - & - & - & - & 0,19 & 0,08 & 0,51 & 0,26 & 88 \\
\hline Tapirira guianensis & - & - & - & - & - & 0,19 & 0,08 & 0,51 & 0,26 & 89 \\
\hline Alchornea glandulosa & - & - & - & - & - & 0,19 & 0,04 & 0,51 & 0,25 & 93 \\
\hline Guatteria sp. & - & - & - & - & - & 0,19 & 0,03 & 0,51 & 0,25 & 100 \\
\hline Remijia sp. & - & - & - & - & - & 0,19 & 0,03 & 0,51 & 0,25 & 102 \\
\hline Xylopia sericea & - & - & - & - & - & 0,19 & 0,03 & 0,51 & 0,25 & 103 \\
\hline Tovomita glazioviana & - & - & - & - & - & 0,19 & 0,03 & 0,51 & 0,25 & 104 \\
\hline Machaerium aculeatum & - & - & - & - & - & 0,19 & 0,03 & 0,51 & 0,25 & 105 \\
\hline Inga sp. & - & - & - & - & - & 0,19 & 0,03 & 0,51 & 0,25 & 109 \\
\hline Rudgea lanceolata & - & - & - & - & - & 0,19 & 0,03 & 0,51 & 0,25 & 112 \\
\hline Total & 100 & 100 & 100 & 100 & - & 100 & 100 & 100 & 100 & - \\
\hline
\end{tabular}

"-_" indica ausência da espécie na listagem, sendo atribuído o valor "zero" para cada espécie para cálculo do teste F de Graybill (1976); DR é a densidade relativa, DoR é a dominância relativa, FR é a frequência relativa e IVI é o índice de valor de importância das espécies.

O modelo em nível de árvore individual mostrou-se preciso na estimação do volume por unidade de área, distribuição diamétrica e de altura e em nível de árvore individual para a Floresta em estudo. Do ponto de vista prático, este modelo confere maior versatilidade no planejamento da produção, uma vez que se pode estimar o volume de madeira para diferentes usos, haja vista que se tem a projeção das dimensões árvore a árvore, inclusive do fuste comercial (uso mais nobre).

Os resultados encontrados confirmam o potencial de utilização de modelos em nível de árvore individual em florestas naturais estruturalmente complexas, como é o caso da Floresta Estacional Semidecidual Montana em estágio médio de sucessão ecológica.

\section{CONCLUSÕES}

O modelo de árvore individual avaliado é eficiente para estudo do crescimento e da produção da Floresta Estacional Semidecidual.

As estimativas foram precisas em todos os níveis de detalhamento: em nível de povoamento total, distribuição diamétrica e altura, e em nível de árvore individual.

Novas investigações devem ser realizadas na tentativa de aprimorar o submodelo de ingresso, sendo necessário, portanto, a coleta de informações da regeneração das florestas (menores níveis de inclusão nos inventários florestais). 


\section{REFERÊNCIAS}

Arce, J. E., Pizatto, W., Sanquetta, C. R., Wendling, J. L. G., \& Maestri, R. (2001). Utilização das matrizes de transição na avaliação e simulação precoces do crescimento de povoamentos de Pinus taeda $\mathrm{L}$. Revista Floresta, 27(1/2), 83-98.

Austregésilo, S. L., Ferreira, R. L. C., Silva, J. A. A., Souza, A. L., Meunier, I. M. J., \& Santos, E. S. (2004). Comparação de métodos de prognose da estrutura diamétrica de uma floresta estacional semidecidual secundaria. Revista Árvore, 28(2), 227-232. http://dx.doi.org/10.1590/S010067622004000200009

Azevedo, C. P., Souza, A. L., \& Jesus, R. M. (1995). Um modelo de matriz de transição para prognose do crescimento de um povoamento natural remanescente não manejado de mata atlântica. Revista Árvore, 19(2), 187-199.

Brasil. Ministério do Meio Ambiente - MMA. (2012). Recuperado em 13 de fevereiro de 2018, de http://www.mma.gov.br

Centro Tecnológico de Minas Gerais - CETEC. (1995). Determinação de equações volumétricas aplicáveis ao manejo sustentado de florestas nativas do estado de Minas Gerais e outras regiões do País (295 p., Relatório Final). Belo Horizonte: Fapemig/CETEC.

Davis, L. S., \& Johnson, K. N. (1987). Forest management (3rd ed., 790 p.). New York: McGraw-Hill.

Freitas, J. V., \& Higuchi, N. (1993). Projeções da distribuição diamétrica de uma floresta tropical úmida de terra firme pela cadeia de Markov. In Anais do Congresso Florestal Panamericano: Floresta para o Desenvolvimento: Política, Ambiente, Tecnologia e Mercado (Vol. 2, pp. 545-548). São Paulo: SBEF, SBS.

Gandolfi, S., Leitão Filho, H. F., \& Bezerra, C. L. (1995). Levantamento florístico e caráter sucessional de espécies arbustivo-arbóreas de uma floresta Mesófila Semidecídua no município de Guarulhos, SP. Revista Brasileira de Biologia, 55(4), 753-767.

Graybill, F. A. (1976). Theory and application of linear model (704 p.). Massachusetts: Belmont Duxbury.

Hasenauer, H. (2000). Princípios para a modelagem de ecossistemas florestais. Ciência \& Ambiente, (20), 53-69.

Menon, U. M., Figueiredo Filho, A., \& Watzlawick, L. F. (2010). Matriz de transição para predição da distribuição diamétrica em Floresta Ombrófila Mista na flona de Irati (PR). Ambiência, 6(2), 271-280.

Monserud, R. A., \& Sterba, H. (1999). Modeling individual tree mortality for Australian forest species. Forest Ecology and Management, 113(2), 109-123. http://dx.doi.org/10.1016/S0378-1127(98)00419-8.

Pretzsch, H., Biber, P., \& Durský, J. (2002). The single tree-based stand simulator Silva: construction, application and evaluation. Forest Ecology and Management, 162(1), 3-21. http://dx.doi.org/10.1016/S0378-1127(02)00047-6.

Pulz, F. A. (1998). Estudo da dinâmica e a modelagem da estrutura diamétrica de uma floresta semidecídua montana na região de Lavras - MG (Dissertação de mestrado). Universidade Federal de Lavras, Lavras.

Pulz, F. A., Scolforo, J. R. S., Oliveira, A. D., Mello, J. M., \& Oliveira Filho, A. T. (1999). Acuracidade da predição da distribuição diamétrica de uma floresta inequiânea com a matriz de transição. Revista Cerne, 5(1), 1-14.

Rossi, L. M. B., Koehler, H. S., Sanquetta, C. R., \& Arce, J. E. (2007). Modelagem de mortalidade em florestas naturais. Revista Floresta, 37(2), 275-291.

Sanquetta, C. R., Brena, D. A., Ângelo, H., \& Mendes, J. B. (1996). Matriz de transição para simulação da dinâmica de florestas naturais sob diferentes intensidades de corte. Ciência Florestal, 6(1), 65-78. http://dx.doi.org/10.5902/19805098326.

Scolforo, J. R. S., Pulz, F. A., Mello, J. M., \& Oliveira Filho, A. T. (1996). Modelo de produção para floresta nativa como base para o manejo sustentado. Revista Cerne, 2(1), 112-137.

Shimizu, J. Y. (2007). Estratégia complementar para conservação de espécies florestais nativas: resgate e conservação de ecótipos ameaçados. Pesquisa Florestal Brasileira, (54), 7-35.

Silva, J. N. M., \& Lopes, J. C. A. (1984). Inventário florestal contínuo em florestas tropicais: a metodologia utilizada pela Embrapa-CPATU na Amazônia brasileira (Documentos, No. 36, 36 p.). Belém: EmbrapaCPATU.

Soares, C. P. B., Paula Neto, F., \& Souza, A. L. (2011). Dendrometria e inventário florestal (2. ed., 272 p.). Viçosa: Editora UFV.

Sokal, R. R., \& Rohlf, F. J. (1969). Biometry: the principles and practice of statistics in biological research $(776$ p.). San Francisco: Freeman and Company. 
SOS Mata Atlântica. (2012). Mata Atlântica. São Paulo. Recuperado em 12 de fevereiro de 2018, de http://www.sosmatatlantica.org.br

Stepka, T. F., Dias, A. N., Figueiredo Filho, A., Machado, S. A., \& Sawczuk, A. R. (2010). Prognose da estrutura diamétrica de uma Floresta Ombrófila Mista com os métodos razão de movimentos e matriz de transição. Pesquisa Florestal Brasileira, 30(64), 327-335. http://dx.doi.org/10.4336/2010.pfb.30.64.327.

Taylor, S. L., \& Maclean, D. A. (2007). Spatiotemporal patterns of mortality in declining balsam fir and spruce stands. Forest Ecology and Management, 253(1), 188-201. http://dx.doi.org/10.1016/j.foreco.2007.07.016.

Teixeira, L. M., Chambers, J. Q., Silva, A. R., Lima, A. J. N., Carneiro, V. M. C., Santos, J., \& Higuchi, N. (2007). Projeção da dinâmica da floresta natural de Terra-firme, região de Manaus-AM, com o uso da cadeia de transição probabilística de Markov. Acta Amazonica, 37(3), 377-384. http://dx.doi.org/10.1590/S0044-59672007000300009.

Tomé, M. (1991). Os modelos de produção florestal na gestão de povoamentos florestais. Revista Agros, $8(2), 32-36$.

Vanclay, J. K. (1994). Modeling forest growth and yield: aplications to mixed tropical forest (312 p.). Wallingford: CAB International.

Contribuição dos autores: RVOC: conceituação, análise formal, obtenção de financiamento, investigação, metodologia, administração do projeto, supervisão, validação, escrita - primeira redação; CPBS: conceituação, curadoria dos dados, investigação, metodologia, administração do projeto, supervisão, escrita - revisão e edição; HGL: conceituação, investigação, metodologia, supervisão, escrita - revisão e edição; ALS: conceituação, curadoria dos dados, investigação, metodologia, escrita - revisão e edição; FBM: metodologia, escrita - revisão e edição; GSN: escrita - revisão e edição, MLRO: escrita - revisão e edição. 